\title{
Theory of reverse combustion linking
}

\author{
M.S. Blinderman \\ Ergo Exergy Technologies Inc., CANADA \\ A.Y. Klimenko ${ }^{1}$ \\ School of Engineering, University of Queensland, AUSTRALIA
}

\begin{abstract}
The present work suggests a theory of Reverse Combustion Linking (RCL). RCL is a central unit operation of Underground Coal Gasification (UCG) technology. The theory is based on analysing stability of different branches of the propagation speed curves and determining the regime that is responsible for propagation of the flame during RCL. The theory is in a good qualitative and quantitative agreement with the data obtained in practical use of RCL in UCG operations.
\end{abstract}

Key words: Undeground Coal Gasification, Reverse Combustion Linking

\section{Introduction}

Reverse combustion linking (RCL) is a key operation of Underground Coal Gasification (UCG, $[1,2]$ ). The term "reverse combustion linking " became conventional in UCG applications and we follow this convention in the present work. UCG is a gasification process carried on in non-mined coal seams using injection and production wells drilled from the surface, which enables the coal to be converted into product gas. RCL is a method of linking the process wells within a coal seam, which includes injection of an oxidant into one well and ignition of coal in the other so that combustion propagates towards the source of oxidant thereby establishing a low hydraulic resistance path between the two wells as shown in Figure 1. This allows to inject an oxidant and produce synthesis gas at commercially significant flow rates. A procedure to conduct RCL includes the following: an oxidant is injected in one of the wells (injection well) while coal seam is ignited in the other well (production well). Combustion of coal in the production well proceeds towards the source of oxidant. Injection of oxidant into the injection well is maintained until the fire reaches the bottom of the well. Creation of a low hydraulic resistance link between the

$\overline{1}$ corresponding author E-mail: klimenko@mech.uq.edu.au 
bottoms of the process wells results in significant drop in the pressure difference between the wells indicating successful completion of the linking process. RCL is followed by main stages of UCG that are not considered in the present work. RCL is conventionally characterised by the speed of linking measured in meters per day and by oxidant consumption measured in normal cubic meters per hour.

Among a number of publications devoted to reverse combustion in porous media $[1,3-6]$, only the analytical work of Britten and Krantz [1] is specifically devoted to RCL. In this work, Britten and Krantz considered RCL while solving a set of modelling equations for a planar reverse combustion front and focusing their attention on the equations for the gas phase, implicitly assuming deficiency of air and excess of coal in the reaction zone and downstream from it. Although their approach confirmed hydrodynamic instability of the front, which is an important factor in the link formation, the conceptual understanding of RCL employed by Britten and Krantz contradicts empirical evidence. They consider RCL as the process of coal pyrolysis followed by combustion of the released volatile matter so that the hydraulic link between injection and production wells is formed by a porous path of an enhanced permeability. However, empirical data from UCG operations indicate that RCL results routinely in formation of a channel in which coal has been essentially consumed by combustion.

In our approach we, to a large extent, follow the principle formulation of reverse combustion introduced by Schult et al [5] and used in the recent work of Liu et. al. [6]. Unlike the formulation of Britten and Krantz [1], the formulation of Schult et al [5] allows for analysis of both oxigen-deficient and coal deficient flames. We assume, however, as did Britten and Krantz [1], that gas phase in the reaction zone is not dominated by diffusion. We analyse both oxygen-deficient and coal-deficient cases and their hydrodynamic and pulsating stability and pay special attention to the vicinity of the stoichiometric point. As the result of the analysis, we found that the flames close to the stoichiometric regime (as defined for incomplete combustion) are primarily responsible for RCL. Another feature of the present work is consideration of curved flames and their propagation speed, which is important for understanding of RCL. It is treated using Intrinsic Disturbed Flame Equations (IDFE, [7-10]). Although in most of the paper we implicitly follow the developed asymptotic methodologies, at several instances we opted for a simplified (rather than strict asymptotic) formulation that allows to obtain compact analytical representations of the results but does retain the important physical effects that are of primary importance in RCL. This approach resulted in an overall theory of reverse combustion linking during UCG that determines main relationships between key parameters of the process. Preliminary results of applying the theory to specific UCG conditions demonstrate a reasonable conformity with the data obtained in practical UCG operations. 


\section{The modelling equations}

The equations governing transport and reactions in porous media are represented by the conservation of mass, energy and species equations that can be written in the form

$$
\begin{gathered}
\frac{\partial \bar{\rho}}{\partial t}+\nabla \cdot(\overline{\mathbf{m}})=0, \frac{\partial \overline{\rho_{i}}}{\partial t}+\nabla \cdot\left(\overline{\mathbf{m}_{i}}\right)=\overline{W_{i}} \\
\frac{\partial \overline{\rho h}}{\partial t}+\nabla \cdot(\overline{\mathbf{m} h})-\nabla \cdot(\lambda \nabla(T))=0
\end{gathered}
$$

Here, $\overline{\mathbf{m}} \equiv \overline{\rho \mathbf{v}}$ and $\mathbf{m}_{i}=\mathbf{v}_{i} \rho_{i}$ are the mass fluxes, the subscript index " $i$ " runs over all species of interest; $\rho_{i}$ is the density (mass per volume) of the specific component (so that $Y_{i}=\rho_{i} / \rho$ is the mass fraction), $\lambda$ is the effective heat conductivity and $W_{i}$ is the chemical reaction source terms of the i'th component. The mass flux $\rho \mathbf{v}$ is denoted by $\mathbf{m}$. The temporal pressure derivative $\partial p / \partial t$ is conventionally neglected in the energy equation. The over-bar denotes the superficial averages:

$$
\begin{aligned}
\overline{\mathbf{m}} & =\phi_{g} \mathbf{m}_{g}+\phi_{q} \mathbf{m}_{q}+\phi_{s} \mathbf{m}_{s}=\sum_{i} \phi_{i} \mathbf{m}_{i}=\sum_{i} \phi_{i} \rho_{i} \mathbf{v}_{i} \\
\overline{\mathbf{m} h} & =\phi_{g} \mathbf{m}_{g} h_{g}+\phi_{q} \mathbf{m}_{q} h_{q}+\phi_{s} \mathbf{m}_{s} h_{s}=\sum_{i} \phi_{i} \mathbf{m}_{i} h_{i}
\end{aligned}
$$

The superficial averages can be evaluated as the sum over parameters of the three phases - gas, liquid and solid - that are denoted by the subscript indices " $g$ ", " $q$ " and "s". The specific volumes $\phi$ occupied by the phases are determined by the porosity of the solid phase. The superficial averages can also be evaluated as a sum over all species involved. The species are indexed by the running index " $i$ " but we should note that velocities $\mathbf{v}$ and specific volumes $\phi$ are the same for all components of a given phase, although $\mathbf{v}$ and $\phi$ are, generally, different for different phases. For example, $\mathbf{v}_{i}=\mathbf{v}_{g} \neq \mathbf{v}_{s}$ for any " $i$ " that belongs to the gas phase. The averages that are applied to a single component such as $\overline{\mathbf{m}_{i}}=\phi_{i} \mathbf{m}_{i}=\phi_{i} \rho_{i} \mathbf{v}_{i}$ do not involve summation over the other species. Similarly, $\overline{\mathbf{m}_{g}}=\phi_{g} \mathbf{m}_{g}=\phi_{g} \rho_{g} \mathbf{v}_{g}$ for the gas phase and other phases. The temperature $T$ is considered to be the same for all phases and does not need averaging. We do not consider variations of the values in the interparticle space. Hence, the intrinsic averages simply coincide with the values determined within a specific phase and no special notation is needed here for intrinsic averages. The momentum equation is represented by the Darcy law

$$
\mathbf{v}_{g}=\mathbf{U}+\mathbf{S}, \quad \mathbf{U}=-f \nabla p
$$

where $S$ is the velocity associated with the moving frame of reference (i.e. the flame speed) and $f$ is permeability.

In the present analysis we do not consider the liquid phase separately, assuming that the underground water is supplied with the gas flow or represents the coal moisture content. Hence only two phases, gas and solid, need to be indexed explicitly. In terms of the phases the global reaction takes the form

$$
\mu_{g}^{-}(\mathrm{Gas})^{-}+\mu_{s}^{-}(\mathrm{Solid})^{-} \rightarrow \mu_{g}^{+}(\mathrm{Gas})^{+}+\mu_{s}^{+}(\mathrm{Solid})^{+}
$$


or

$$
\sum_{i} \mu_{i}^{-}(\text {Species })_{i} \rightarrow \sum_{i} \mu_{i}^{+}(\text {Species })_{i}
$$

The coefficients $\mu_{i}$ are on the mass basis. The superscripts "-" and "+" denote values before and after reactions and $\mu_{i}^{+}-\mu_{i}^{-}$represents the mass change of $i^{\text {th }}$ component. In the rest of the paper we put $\mu_{g}^{-}=1$ without loss of generality. The global reaction is presumed to be specified so that the products coefficients depend on $\mu_{s}^{-}$so that $\mu_{i}^{ \pm}=\mu_{i}^{ \pm}\left(\mu_{s}^{-}\right)$for any component " $i$ " (or phase " $i$ "). All of the reactions are presumed to form a global reaction that has a single rate so that we can write for any $i$ and $j$

$$
\frac{\overline{W_{i}}}{\mu_{i}^{+}-\mu_{i}^{-}}=\frac{\overline{W_{j}}}{\mu_{j}^{+}-\mu_{j}^{-}}
$$

The total enthalpy $h_{i}$ of each component can be represented as a sum of sensible enthalpy and enthalpy of formation $h_{i}=h_{i}^{(s)}+h_{i}^{(f)}$ with the superscripts " $s "$ and " $f$ " denoting the sensible and formation enthalpies. The enthalpy equation takes the form

$$
\frac{\partial \overline{\rho h^{(s)}}}{\partial t}+\nabla \cdot\left(\overline{\mathbf{m} h^{(s)}}\right)-\nabla \cdot(\lambda \nabla(T))=\overline{W_{h}}
$$

where

$$
\begin{gathered}
-\overline{W_{h}} \equiv \frac{\partial \overline{\rho h^{(f)}}}{\partial t}+\nabla \cdot\left(\overline{\mathbf{m} h^{(f)}}\right)=\sum_{i} h_{i}^{(f)}\left(\frac{\partial \overline{\rho_{i}}}{\partial t}+\nabla \cdot \overline{\mathbf{m}_{i}}\right)=\sum_{i} h_{i}^{(f)} \overline{W_{i}}=q \overline{W_{O 2}}, \\
q \equiv \frac{H\left(\mu_{s}^{-}\right)}{\mu_{O 2}^{-}-\mu_{O 2}^{+}}, \quad H\left(\mu_{s}^{-}\right) \equiv \sum_{i}\left(\mu_{i}^{-}-\mu_{i}^{+}\right) h_{i}^{(f)}
\end{gathered}
$$

and $H\left(\mu_{s}^{-}\right)$is the enthalpy of the reaction. The enthalpy of formation is defined as the enthalpy at a certain reference temperature $T^{(f)}$ whose conventional value is $25^{0} \mathrm{C}$. (The best choice for $T^{(f)}$, which accurately defines the heating value of the reaction, is a constant temperature that is close to the temperature in the reaction zone). Multiplying equations for the each component in (1) by $\left(h_{i}^{(s)}\right)=h_{i}^{(s)}\left(T_{u}\right)$, evaluating the sum over all " $i$ " and subtracting the result from equation (4) yields the equation for the sensible enthalpy increments $\Delta h_{i}^{(s)}$

$$
\frac{\partial \overline{\rho \Delta h^{(s)}}}{\partial t}+\nabla \cdot\left(\overline{\mathbf{m} \Delta h^{(s)}}\right)-\nabla \cdot(\lambda \nabla(T))=\overline{W_{h}}+\overline{W_{h}^{(s)}}
$$

where

$$
\Delta h_{i}^{(s)} \equiv h_{i}^{(s)}-\left(h_{i}^{(s)}\right)_{u} ; \quad W_{h}^{(s)} \equiv-\sum_{i} \overline{W_{i}}\left(h_{i}^{(s)}\right)_{u}
$$

The additional term $W_{h}^{(s)}$ can be nullified by selecting $T^{(f)}=T_{u}$. 


\section{Planar flame}

A simple, planar flame front propagating under stationary conditions is characterised by the following system of equations

$$
\begin{gathered}
\frac{\partial \bar{m}}{\partial x}=0, \frac{\partial \overline{m_{i}}}{\partial x}=\overline{W_{i}} \\
\frac{\partial \overline{m h}}{\partial x}-\frac{\partial}{\partial x}\left(\lambda \frac{\partial T}{\partial x}\right)=0
\end{gathered}
$$

Here, we use the system of coordinates that is attached to the flame; for the first components of vectors that is normal to the flame front $-x^{(1)}, v^{(1)}, m^{(1)}$ and others - the index identifying the normal component is omitted here and further in the article. Integration of these equations across the flame yields

$$
\begin{gathered}
(\bar{m})_{b}=(\bar{m})_{u} \\
\left(\overline{m_{i}}\right)_{b}=\left(\overline{m_{i}}\right)_{u}+Q_{i}, \quad Q_{i} \equiv \int_{-\infty}^{+\infty} \overline{W_{i}} d x \\
(\overline{m h})_{b}=(\overline{m h})_{u}
\end{gathered}
$$

The subscript " $u$ " is used to denote values upstream from the flame while the subscript "b" denotes values downstream from the flame front. Equations (4) and (11) can be written as

$$
\begin{gathered}
\frac{\partial \overline{m h^{(s)}}}{\partial x}-\frac{\partial}{\partial x}\left(\lambda \frac{\partial T}{\partial x}\right)=\overline{W_{h}} \\
\left(\overline{m h^{(s)}}\right)_{b}=\left(\overline{m h^{(s)}}\right)_{u}+Q_{h}
\end{gathered}
$$

where equations (5) indicate that

$$
Q_{h} \equiv \int_{-\infty}^{+\infty} \overline{W_{h}} d x=\left(\overline{m h^{(f)}}\right)_{u}-\left(\overline{m h^{(f)}}\right)_{b}=\left(\overline{m_{g}}\right)_{u} H\left(\mu_{s}^{-}\right)
$$

\subsection{The reaction zone}

The major reaction in the global reaction mechanism is oxidation of carbon

$$
\mathrm{C}+(1-n / 2) \mathrm{O}_{2} \rightarrow n \mathrm{CO}+(1-n) \mathrm{CO}_{2}
$$

that is expected to be incomplete when $n<1$. The reaction machanism may also include other reactions and phase transitions (evaporation of water, for example, has a significant effect on the flame temperature), although the rate of the major reaction is presumed to determine all other reactions and phase transitions. The rate of the major reaction that determines consumption of oxygen is given by

$$
\overline{W_{O 2}}=-\varphi\left(\overline{\rho_{C}}\right) A \rho_{O 2} K(T) ; \quad \frac{1}{K(T)}=\frac{1}{K_{r}(T)}+\frac{1}{K_{t}}, \quad K_{r}(T)=\exp \left(-T_{E} / T\right)
$$


For temperatures lower than $T_{t}$ specified by $K_{t}=\exp \left(-T_{E} / T_{t}\right)$, the rate is reactionlimited $K \approx K_{r}$ and, for the temperatures higher than $T_{t}$, the rate is transport-limited $K \approx K_{t}$. The reaction parameter $A$ is presumed constant while $\varphi=1$ when $\overline{\rho_{C}}>0$ and $\varphi=0$ when $\overline{\rho_{C}}=0$. Assuming that the reaction zone is thin in comparison with the flame thickness, we obtain

$$
\begin{aligned}
& \frac{\partial \bar{m}}{\partial x}=0, \frac{\partial \overline{m_{i}}}{\partial x}=\overline{W_{i}} \\
& -\frac{\partial}{\partial x}\left(\lambda_{r} \frac{\partial T}{\partial x}\right)=\overline{W_{h}}
\end{aligned}
$$

The integral of the enthalpy equation is given by

$$
\left(\lambda T^{\prime}\right)_{(-r)}=Q_{h}=\left(\overline{m h^{(f)}}\right)_{(-r)}-\left(\overline{m h^{(f)}}\right)_{b}
$$

The subscript " $r$ " is used to denote the values related to the reaction zone, for example, $\lambda_{r}$ is the diffusion coefficient within reaction zone that, conventionally, can be assumed to be constant $\lambda_{r}=\lambda_{b}$ since the reaction zone is presumed thin. The subscript and superscript indices "-" are used to emphasise that the indexed values are related to the reactants while the index "+" points to the products. To make equations concise, we introduced $T^{\prime} \equiv \partial T / \partial x$. The reaction rate for the heat release is linked to the reaction rate by $q \overline{W_{O 2}}=-\overline{W_{h}}$ where the coefficient $q=q\left(\mu_{s}^{-}\right)=H\left(\mu_{s}^{-}\right) /\left(\mu_{O 2}^{-}-\mu_{O 2}^{+}\right)$represents heat released by oxidation with one unit of mass of $\mathrm{O}_{2}$. Hence, with the use of (15) we may write

$$
\frac{\partial \lambda_{r} T^{\prime}}{\partial x}=q \frac{\partial \phi_{g} v_{g} \rho_{O 2}}{\partial x}=-q \varphi A \rho_{O 2} K(T)
$$

Since $\left(T^{\prime}\right)_{(+r)}=\left(T^{\prime}\right)_{b}=0$, we use the following linear dependence between $T^{\prime}$ and $\rho_{O 2}$

$$
\rho_{O 2}=\rho_{O 2}\left(T^{\prime}\right)=\left(\rho_{O 2}\right)_{b}+\left(\rho_{O 2}\right)_{\Delta} \frac{T^{\prime}}{\left(T^{\prime}\right)_{(-r)}}
$$

Here and further in the paper we denote $(\cdot)_{\Delta} \equiv\left|(\cdot)_{u}-(\cdot)_{b}\right|$. Linear function (20) represents an exact integral if $\phi_{g} v_{g}=$ const through the reaction zone and this is the case when the reactions do not change number of moles in the gaseous phase (for example, $\mathrm{C}+\mathrm{O}_{2} \rightarrow \mathrm{CO}_{2}$ ) in other cases the linear dependence represents an approximation that is physically plausible and, at the same time, ensures reasonably simple integration of (19). Multiplying (19) by $T^{\prime}$ and separating variables leads to

$$
\frac{\lambda_{r}}{q \varphi A} \frac{T^{\prime} d T^{\prime}}{\rho_{O 2}\left(T^{\prime}\right)}=-K(T) d T
$$

Evaluating of the integrals

$$
\begin{gathered}
\int_{0}^{T_{(-r)}^{\prime}} \frac{T^{\prime} d T^{\prime}}{\rho_{O 2}\left(T^{\prime}\right)}=\frac{\left(T_{(-r)}^{\prime}\right)^{2}}{\left(\rho_{O 2}\right)_{\Delta}}(1-\xi) \\
\Phi\left(T_{b}\right)=\int_{-\infty}^{T_{b}} K(T) d T=\frac{K_{t}}{Z} \ln \left(1+\frac{K_{r}\left(T_{b}\right)}{K_{t}}\right)
\end{gathered}
$$


where

$$
\xi \equiv \frac{\left(\rho_{O 2}\right)_{b}}{\left(\rho_{O 2}\right)_{\Delta}} \ln \left(\frac{\left(\rho_{O 2}\right)_{u}}{\left(\rho_{O 2}\right)_{b}}\right), \quad Z \equiv \frac{T_{E}}{T_{b}^{2}}
$$

is performed by expanding the exponent $\exp \left(-T_{E} / T\right)=\exp \left(-T_{E} / T_{0}+Z\left(T-T_{0}\right)\right)$ in vicinity of a certain reference temperature $T_{0}$ that is selected close to $T_{b}$. In evaluations of the RCL rates presented below we assume $T \ll T_{t}$, hence $K_{r} \ll K_{t}$ and $\Phi\left(T_{b}\right)=K_{r}\left(T_{b}\right) / Z$. Finally, we note that $Q_{h}=q Q_{O 2}$ and obtain

$$
Q_{h}\left(T_{b}, \ldots\right)=\lambda_{r} T_{(-r)}^{\prime}=\sqrt{\frac{\left(\rho_{O 2}\right)_{\Delta} q A \lambda_{r}}{1-\xi} \Phi\left(T_{b}\right)}
$$

\subsection{The planar flame speed}

The speed of the planar flame can be obtained without specific analysis of the preheat zone. We first note that the reaction coefficients are linked to the mass fluxes by the equations

$$
\mu_{g}^{-}=1, \mu_{i}^{+}=\frac{\left(\overline{m_{i}}\right)_{b}}{\left(\overline{m_{g}}\right)_{u}}, \mu_{i}^{-}=\frac{\left(\overline{m_{i}}\right)_{u}}{\left(\overline{m_{g}}\right)_{u}}
$$

for any $i$ representing different species or phases. Equation (11) divided by $\left(\overline{m_{g}}\right)_{u}$ indicates that the enthalpy is preserved

$$
\sum_{i} \mu_{i}^{+} h_{i}\left(T_{b}\right)=\sum_{i} \mu_{i}^{-} h_{i}\left(T_{u}\right) \Longrightarrow T_{b}=T_{a}\left(\mu_{s}^{-}, \ldots\right)
$$

This determines the temperature of the products $T_{b}$ as the adiabatic temperature $T_{a}$ for given fuel/air ratio $\mu_{s}^{-}$. Dependence of $T_{b}$ on $\mu_{s}^{-}$for the conditions analysed in Section 6 is shown in Figure 2. Equation (14) determines the gas and solid mass flow rates

$$
\left(\overline{m_{g}}\right)_{u}=\frac{Q_{h}\left(T_{b}, \ldots\right)}{H\left(\mu_{s}^{-}\right)}, \quad\left(\overline{m_{s}}\right)_{u}=\mu_{s}^{-}\left(\overline{m_{g}}\right)_{u}
$$

and the flame and gas velocities

$$
S=v_{s}=\frac{\left(\overline{m_{s}}\right)_{u}}{\left(\phi_{s} \rho_{s}\right)_{u}}, \quad\left(v_{g}\right)_{u}=U_{u}+S=\frac{\left(\overline{m_{g}}\right)_{u}}{\left(\phi_{g} \rho_{g}\right)_{u}}
$$

The flame speed strongly depends on the temperature of the products $T_{b}$ which, in its turn, strongly depends on fuel/air ratio $\mu_{s}^{-}$. The functions $T_{a}\left(\mu_{s}^{-}, \ldots\right)$ and $Q_{h}\left(T_{b}, \ldots\right)$ also depend on other parameters such as $T_{u}, A, T_{E}$ and initial compositions of coal and air that are fixed and selected to match the conditions in the coal seam.

The dependence of the flame speed $S$ on the gas velocity in the fresh mixture $U_{u}$ is shown in Figure 3. In Figures 2 and 3, the dashed curve corresponds to irreversible reactions with a jump in the slope of the curve at point 5 that corresponds to the stoichiometric air/fuel ratio (for a given proportion $n$ between $\mathrm{CO}$ and $\mathrm{CO} 2$ in the reaction products). This jump is induced by the jump in the slope of the adiabatic temperature function $T_{a}\left(\mu_{s}^{-}\right)$. Two propagation speeds correspond to the upper and lower branches of the curve and a single 
given value of $U_{u}$. The upper branch of the curve is oxygen-deficient and it is hotter than the lower branch that is coal-deficient. The temperatures near the point 0 are low, the flame speed is small and the flame becomes practically extinct. The flame can not be sustained and is blown off by any flow with a gas velocity that is higher than $\left(U_{u}\right)_{5}$ (i.e $U_{u}$ at point 5) The stability analysis presented in the appendix indicates that the lower branch is unstable due to pulsating instability. The flames that correspond to this branch either rapidly accelerate and reach the upper branch or decelerate and become extinct. The role of this branch is more in separating the flames that are sustainable from the flames that are extinguished. The upper branch is also not stable - it is affected by the hydrodynamic instability that is less severe than the pulsating instability. Our assessment of the stability of RCL is, generally, in agreement with the other works on reverse combustion $[1,5,6,11]$. The details of the stability analysis are given in the Appendix.

As we show in the next sections, the vicinity of the stoichiometric point 5 in Figure 3 plays a major role in RCL but the dashed curve does not allow to analyse the details of the stoichiometric point. In a more realistic case, $T_{a}\left(\mu_{s}^{-}\right)$appears to be smoothed near the stoichiometric point and this case corresponds to the solid curves in Figures 2 and 3. One can notice that instead of the single maximum velocity point, we now have two maximum points: point 2 where the flame speed $S$ reaches its maximum $(S)_{2}$ (and $B_{U}=0$ in (A.5)) and point 3 that corresponds to the maximal value of the gas velocity $\left(U_{u}\right)_{3}$ (and $B_{S}=0$ in (A.5)). The stability analysis shows that the branch between points 0 and 2 is hydrodynamically unstable, the branch between points 3 and 0 is unstable due to pulsating instability while the section of the curve between points 2 and 3 is stable. The vicinity of point 2 can be considered as practically stable due to obvious physical limitations imposed on the scale of the disturbances so that $k$ in equation (A.19) of the appendix can not be infinitely small. Point 3 represents the most stressed regime of the flame separating the sutained flame from the flame blow-off and point 3 is therefore unstable for practical purposes.

\section{Disturbed flame}

The Intrinsic Disturbed Flame Equations (IDFE) is an asymptotic methodology of analysing structure of the flame fronts curved and stretched by surrounding flow. IDFE were first introduced in Ref [7]. The main features of IDFE is using specially selected adaptive curvilinear system of coordinates attached to the flame that radically simplifies formulation of the problem. In curvilinear system of coordinates, differential equations involve $g^{2}$ - the determinant of the metric tensor that defines local metrics of the curvilinear coordinates. The IDFE coordinates are selected so that $g$ is proportional to the area of a flame surface segment. As discussed further in this section, the value of $g$ is determined by two major parameters: the spatial derivative of $g$, which is related to flame curvature $\gamma$, and the temporal derivative of $g$, which is related to the flame stretch $\kappa$ The most useful feature of IDFE is that only two parameters characterising flame/flow interactions ( $\gamma$ and $\kappa$ ) enter the transport equations from early stages of problem formulations. Several results have been obtained with the use of IDFE technique. If any multistep reaction kinetics occurs in 
a zone, which is thin in comparison with the flame width, then $\gamma$ does not explicitly appear in the disturbed flame speed equation [8] but, if the reaction zone is thick, $\gamma$ is, generally, present in the flame speed equation [9]. IDFE have been used to formulate a unifying approach to determining the speed of disturbed flames $[7,10]$, to establish effective flame jump conditions for scalars and velocities [10] and to analyse stability of premixed flames $[12]$.

Here we use IDFE to take into account the effects of flame stretch and curvature as well as some of unsteady effects. In a general curvilinear system of coordinates the transport equations (1), (2) and (4) take the form

$$
\begin{gathered}
\frac{\partial g \bar{\rho}}{\partial t}+\frac{\partial g \bar{m}^{j}}{\partial x^{j}}=0 \\
\frac{\partial g \overline{\rho_{i}}}{\partial t}+\frac{\partial g \bar{m}_{i}^{j}}{\partial x^{j}}=g \overline{W_{i}} \\
\frac{\partial g \overline{\rho h}}{\partial t}+\frac{\partial g \overline{m^{j} h}}{\partial x^{j}}-\frac{\partial}{\partial x^{j}}\left(g^{j k} g \lambda \frac{\partial T}{\partial x^{k}}\right)=0 \\
\frac{\partial g \overline{\rho \Delta h^{(s)}}}{\partial t}+\frac{\partial g \overline{m^{j} \Delta h^{(s)}}}{\partial x^{j}}-\frac{\partial}{\partial x^{j}}\left(g^{j k} g \lambda \frac{\partial T}{\partial x^{k}}\right)=g \overline{W_{h}}+g \overline{W_{h}^{(s)}}
\end{gathered}
$$

where $g=\left|\operatorname{det} g^{j k}\right|^{-1 / 2}, g^{j k}$ is the metric tensor of the of coordinates, the superscript indices run over spatial components of the vectors $j, k=1,2,3$ and the summation convention is applied to repeated indices. The most convenient choice of the coordinate system used in IDFE enforces that $x^{1}$ is perpendicular to the flame (so that $g^{12}=g^{13}=0$ ); $x^{1}$ has the same scaling as the physical coordinate $g_{11}=g^{11}=1$ and the coordinate system is attached to the flame so that $x^{1}=0$ determines the flame (i.e. reaction zone) location $[7,8]$. In the rest of the paper $x^{1}$ is denoted simply as $x$. The operator $\nabla_{f}$ denotes the 2-dimensional divergence evaluated on the flame surface, for example $\nabla_{f} \cdot(g \overline{\mathbf{m}}) \equiv \partial\left(g \bar{m}^{\alpha}\right) / \partial x^{\alpha}$, where $\alpha=2,3$ runs over the coordinates on the flame surface. Although in the case of a single phase the 2-dimensional divergence term can be removed by selecting the proper stretch rate for the flame surface coordinates $x^{2}$ and $x^{3}[7,8]$, the multiphase case requires explicit specification of the surface divergence since the rate can be different for different phases. The transport equations can now be written as

$$
\begin{gathered}
\frac{\partial g \bar{\rho}}{\partial t}+\nabla_{f} \cdot(g \overline{\mathbf{m}})+\frac{\partial g \bar{m}}{\partial x}=0 \\
\frac{\partial g \overline{\rho_{i}}}{\partial t}+\nabla_{f} \cdot\left(g \overline{\mathbf{m}_{i}}\right)+\frac{\partial g \overline{m_{i}}}{\partial x}=g \overline{W_{i}} \\
\frac{\partial g \overline{\rho h}}{\partial t}+\nabla_{f} \cdot(g \overline{\mathbf{m} h})+\frac{\partial g \overline{m h}}{\partial x}-\frac{\partial}{\partial x}\left(g \lambda \frac{\partial T}{\partial x}\right)-\ldots=0 \\
\frac{\partial g \overline{\rho \Delta h^{(s)}}}{\partial t}+\nabla_{f} \cdot\left(g \overline{\mathbf{m} \Delta h^{(s)}}\right)+\frac{\partial g \overline{m \Delta h^{(s)}}}{\partial x}-\frac{\partial}{\partial x}\left(g \lambda \frac{\partial T}{\partial x}\right)-\ldots=g \overline{W_{h}}+g \overline{W_{h}^{(s)}}
\end{gathered}
$$

The triple dots indicate neglecting tangential heat fluxes that are small in thin flames (the major tangential heat losses are taken into account by using the intrinsic curvilinear 
system of coordinates $[7,8]$ ). Integration of equation (37) across the flame results in

$$
\left(\overline{m\left(h^{(s)}-h_{u}^{(s)}\right)}\right)_{(+r)}=Q_{h}-\sum_{i} Q_{i}\left(h_{i}^{(s)}\right)_{u}-Q_{l}
$$

where

$$
Q_{l} \equiv \frac{1}{g_{r}} \int_{-\infty}^{x_{(+r)}}\left(\frac{\partial g \overline{\rho \Delta h^{(s)}}}{\partial t}+\nabla_{f} \cdot\left(g \overline{\mathbf{m} \Delta h^{(s)}}\right)\right) d x
$$

is the rate of heat loss, $g=g_{r}$ is $x$-independent within the reaction zone (but not everywhere within the flame)

$$
\int_{-\infty}^{x_{(+r)}} g \overline{W_{i}} d x=g_{r} Q_{i}
$$

and the values $Q_{i}$ are the same as defined in (10). The subscript " $+r$ " indicates the point at the end of the reaction zone rather than any point in the burned mixture. This distinction is needed since $\bar{m}$ is not necessarily a constant in the burned region due to possible variations of $g$. The reaction zone is thin and, to the leading order, is not affected by the disturbances and the unsteady effects so that, within the reaction zone, the function $g=g(x, t)$ can be replaced by a single value $g_{r}(t)$. We also need to introduce the projected mass fluxes $m_{i}^{*}$ that coincide with $m_{i}$ within the reaction zone, but $\overline{m_{i}^{*}}$ remain constant outside the reaction zone. Thus the projected mass fluxes can be defined as satisfying $\partial \overline{m_{i}^{*}} / \partial x=W_{i}$ so that $\left(\overline{m_{i}^{*}}\right)_{b}-\left(\overline{m_{i}^{*}}\right)_{u}=Q_{i}$. Multiplying the last equation by $h_{i}^{(f)}$ and evaluating the sum over all $i$ results in the relationship

$$
\left(\overline{m^{*} h^{(f)}}\right)_{b}-\left(\overline{m^{*} h^{(f)}}\right)_{u}=Q_{h}=\left(\overline{m_{g}^{*}}\right)_{u} H\left(\mu_{s}^{-}\right)
$$

that is similar to (14). The equation for the sensible enthalpy is, however, directly affected by the flame stretch: with the use of the projected mass fluxes, equation (38) can be written as

$$
\left(\overline{m^{*} h^{(s)}}\right)_{b}-\left(\overline{m^{*} h^{(s)}}\right)_{u}=Q_{h}-Q_{l}
$$

The difference of (42) and (41) represents the equation for the total enthalpy

$$
\left(\overline{m^{*} h}\right)_{b}-\left(\overline{m^{*} h}\right)_{u}=-Q_{l}
$$

so that

$$
\sum_{i} \mu_{i}^{+} h_{i}\left(T_{b}\right)=\sum_{i} \mu_{i}^{-} h_{i}\left(T_{u}\right)-\frac{Q_{l}}{\left(\overline{\left.m^{*}\right)_{u}}\right.}
$$

and

$$
T_{b}=T_{a}\left(\mu_{s}^{-}, \ldots\right)-\delta T_{l}, \quad \delta T_{l}=\frac{Q_{l}}{\left(\overline{m^{*} C_{p}}\right)_{b}}
$$

where the last equation is based on the assumption that a constant heat capacity can be used to characterise the mixture between the actual post-burn temperature $T_{b}$ and the adiabatic temperature $T_{a}$. The heat release rate is determined by equation (25), which is not affected by the stretch due to thinness of the reaction zone, and, for given $Q_{h}$, the flame speed is determined by (41) in accordance with (29).

The asymptotic value of the integral $Q_{l}$ defined by (39) is now evaluated. This integral is considered to be a correction so that it can be represented as a sum of the terms and each 
of this terms is linear with respect to the cause of the heat loss. The non-linear terms are smaller than the leading linear terms and are therefor neglected. It should be noted that small variations of the temperature $T_{b}$ can result in significant changes of $Q_{h}\left(T_{b}, \ldots\right)$ and $S$ due to a high activation energy of the reaction. First we expand $g=g_{r}\left(1-\gamma_{0} x+\kappa_{0} t\right)+\ldots$ where

$$
\gamma_{0}=-\left(\frac{\partial \ln g}{\partial x}\right)_{r}, \kappa_{0}=\left(\frac{\partial \ln g}{\partial t}\right)_{r}
$$

are the flame curvature and the flame stretch, evaluated at the surface of the most intense reactions. If the surface coordinates do not expand, we can distinguish the overall stretch $\kappa_{0}$ from the "phase-specific" stretches $\kappa_{i}$

$$
\kappa_{0}=S \gamma_{0}, \quad \kappa_{i}=\nabla_{f} \cdot\left(\mathbf{v}_{i}\right)
$$

The stretch $\kappa_{0}$ is induced by the flame curvature $\gamma_{0}$ and is the same for all phases while $\kappa_{i}$ are phase-specific and occur when the flow of a particular phase (or mixture component) diverges on the flame surface. Within the preheat zone, $\kappa_{i}$ is assumed constant (i.e. permeability and absolute viscosity determining $f$ in Darcy's law are presumed to be temperature-independent). The unsteady effects (which would result in replacement of $\kappa_{0}$ by $\left.\kappa_{0}+d / d t\right)$ are considered separately in Appendix and neglected here. Since $h_{i}^{(s)}$ does not depend, to the leading order, on the surface coordinates $x^{2}$ and $x^{3}$, equation (39) takes the form

$$
Q_{l} \equiv \int_{-\infty}^{x_{r}}\left(\kappa_{0} \overline{\rho \Delta h^{(s)}}+\overline{\kappa \rho \Delta h^{(s)}}\right) d x=\overline{\left(\kappa_{0}+\kappa\right) I}=\sum_{i}\left(\kappa_{0}+\kappa_{i}\right) \phi_{i} I_{i}
$$

where

$$
I_{i}=\int_{-\infty}^{x_{r}} \rho_{i} \Delta h_{i}^{(s)} d x=\int_{T_{u}}^{T_{b}} \frac{\rho_{i} \Delta h_{i}^{(s)} \lambda}{\overline{m^{*} \Delta h^{(s)}}} d T
$$

The integrals $I_{i}$ are mainly dependent on conditions in the preheat zone and, since the reaction zone is thin, can be evaluated with the upper limit just before or just after the reaction zone, whichever approach is more convenient. In the preheat zone where $W_{h}=0$, as it follows from equation (12) the link between the differentials $d T$ and $d x$ is given by $\lambda d T=\overline{m^{*} \Delta h^{(s)}} d x$. Estimations of the integrals $I_{i}$ result in the following expression for the temperature loss

$$
\frac{\delta T_{l}}{T_{\Delta}}=\gamma_{0} l_{f} \theta_{s}+\kappa_{g} \frac{l_{f} \theta_{g}}{U_{u}},
$$

where

$$
\theta_{s} \approx \frac{\left(\overline{m_{s}^{*}\left(C_{p}\right)_{s}}\right)_{u}}{\left(\overline{m^{*} C_{p}}\right)_{u}}, \quad \theta_{g} \approx \frac{\left(\overline{m_{g}^{*}\left(C_{p}\right)_{g}}\right)_{u}}{\left(\overline{m^{*} C_{p}}\right)_{u}}, \quad l_{f} \equiv \frac{\int_{-\infty}^{x_{r}}\left(T-T_{u}\right) d x}{T_{b}-T_{u}} \approx \frac{\lambda_{r}}{\overline{\left(m C_{p}\right)_{u}}}
$$

and $T_{\Delta}=T_{b}-T_{u}$. In these estimations we assume constant heat capacities and put $\left(\overline{m^{*} C_{p}}\right)_{u} \approx\left(\overline{m^{*} C_{p}}\right)_{b}$. This assumption is valid as long as the heat effect of the reaction does not depend strongly on the temperature. The flame thickness can be defined differently for different components $l_{i}=I_{i} /\left(\rho_{i} \Delta h_{i}^{(s)}\right)_{b}$ but any $l_{i}$ can be approximated by $l_{f}$. 


\section{$5 \quad$ Reverse combustion linking}

In order to understand the complexity of the RCL process we first note that the planar reverse combustion flame would be very difficult (and probably impossible) to sustain. Indeed, there is only a small region between points 2 and 3 that can correspond to this regime and be stable. The gas velocity $U_{u}$ must be homogeneous and accurately controlled so that $\left(U_{u}\right)_{2}<U_{u}<\left(U_{u}\right)_{3}$. Practically, any noticeable increase in $U_{u}$ will lead to the flame blow off while any decrease in $U_{u}$ will cause development of instabilities curving the flame.

Similar consideration can be applied to the reverse combustion in a channel that would be inevitably formed due to the instabilities even if the initial conditions correspond to a planar flame. The gas velocity in the channel can't be much greater than $\left(U_{u}\right)_{2}$ (and never exceeds $\left.\left(U_{u}\right)_{3}\right)$, since the flame would be blown off, or noticeably smaller than $\left(U_{u}\right)_{2}$, since this would leave too much space for development of instabilities that would effectively narrow the channel. The channel width that, for a given total mass flow of the gas phase $M_{g}$, corresponds to gas velocity of $\left(U_{u}\right)_{2}$ is the width that would be "preferred" by the process. Under these conditions, the flame can be still treated as oxygen-deficient but nearly all coal within the channel is oxidised in the process. We, however, find a new complication: the speed of $\left(U_{u}\right)_{2}$ is not a constant and would in its turn depend on the channel width. A narrower channel has a more significant heat loss and a lower temperature in the reaction zone and this results in lower $\left(U_{u}\right)_{2}$ and $S_{2}$. This loss would occur at least because the flame in the channel has a convex shape and, in accordance with the IDFE analysis of the previous section, a lower propagation speed. There are also other possible reasons for the temperature decrease in narrow channels (such as ground water influx from coal around the channel and lateral heat loss). Greater $M_{g}$ corresponds to wider channels and greater flame speeds. Very large $M_{g}$ corresponds to minimal losses and any further increase in $M_{g}$ would not speed up the flame but only widen the channel. An insufficient $M_{g}$ results in excessive heat loss and flame extinction.

In reality, the RCL process is even more complicated. It is inevitable that the quality and porosity of coal in the seam varies from point to point resulting in variations of the propagation speed. Let us assume that the channel radius $R_{c}$ is somewhat above the "preferred" value so that $U_{u}$ is below $\left(U_{u}\right)_{2}$. Due to hydrodynamic instabilities, this would result in development of a small cavity. The tip of the cavity, called the leading point, has a radius $R_{p}$ that is smaller than $R_{c}$ while the local velocity at the tip is $\left(U_{u}\right)_{2}$. The leading point propagates faster and is followed by sloping conical flame whose normal propagation velocity is below that of the leading point. The ratio $R_{p} / R_{c}$ can not be too small since this would result in excessively high gas velocities at the leading point and a slow propagation speed or extinction of the tip. If the leading point propagates forward too fast the cavity becomes deep and narrow and this attracts additional gas flow through the leading point so that gas velocity exceeds $\left(U_{u}\right)_{2}$. The leading point is then slowed down and forced to wait until combustion behind the leading point widens the cavity. The leading point can also be extinguished by excessive local gas velocity or other unfavourable conditions. As the rest of the channel catches up with the leading point, the front surface of the channel flattens until conditions favourable for the development of a new leading point 
are attained. It should be noted that not only one but several leading points competing for the gas flow can develop at the same time. The leading point that is closer to the source of the air and/or has better local conditions for the flame propagation is more likely to "win" and lead further channel formation.

Compared to the reverse combustion in a planar flame, the RCL process in a channel has an important stabilising mechanism. If the gas velocity is close to or somewhat above $\left(U_{u}\right)_{3}$, the propagation of the flame is arrested but the flame in the channel is not completely blown off: continuing burning behind the leading point would widen the channel until the gas velocity drops to a more acceptable level. An excessively high $\left(U_{u}\right)_{3}$ can still terminate RCL. If the channel is too wide and $\left(U_{u}\right)_{3}$ is too small for given $M_{g}$, the rapidly advancing leading point would make the following sections of the channel narrower so that $\left(U_{u}\right)_{3}$ in these sections becomes higher.

Assuming that linking forms a channel whose radius is $R_{c}$ and since $S \ll U_{u}$ we may write

$$
\left(\overline{m_{g}}\right)_{u}=\frac{M_{g}}{\pi R_{c}^{2}}
$$

The temperature loss $\delta T_{l}=T_{a}-T_{b}$ appears mainly due to the flame curvature $\gamma_{0}$ at the leading point $\gamma_{0}=2 / R_{p}=2 \zeta / R_{c}$ where $R_{c}$ and $R_{p}$ are connected by a constant factor $\zeta$ that must be not less than unity: $R_{c}=\zeta R_{p}$. Since RCL is close to the stoichiometric regime and coal is fully consumed (or nearly fully consumed) within the forming channel, the gas velocity is directed perpendicular to the flame and $\kappa_{g}=0$ does not contribute to the flame stretch. Hence, equation (50) yields

$$
T_{b}=T_{a}\left(\mu_{s}^{-}\right)-2 \frac{\theta T_{\Delta} l_{f}}{R_{p}}=T_{a}\left(\mu_{s}^{-}\right)-2 \zeta \theta T_{\Delta} l_{f} \sqrt{\pi \frac{\left(\overline{m_{g}}\right)_{u}}{M_{g}}}
$$

At the leading point, the mass flow rates and the flame speed satisfy the following

$$
\left(\overline{m_{g}}\right)_{u} H\left(\mu_{s}^{-}\right)=Q_{h}\left(T_{b}, \ldots\right),\left(\overline{m_{s}}\right)_{u}=\left(\overline{m_{g}}\right)_{u} \mu_{s}^{-}, S=\frac{\left(\overline{m_{s}}\right)_{u}}{\left(\phi_{s} \rho_{s}\right)_{u}}
$$

According to the previous analysis, the $S$ - the velocity of propagation of the leading point - is treated as the value estimating the overall linking speed.

\section{RCL in Chinchilla UCG operations}

The UCG process developed and practiced by Ergo Exergy Technologies is called Exergy UCG or $\varepsilon$ UCG. $\varepsilon U C G$ was applied in the Chinchilla site near Brisbane (Australia) owned by LINC Energy Ltd. (Blinderman and Jones (2002)). RCL has been one of the methods applied in Chinchilla Project to achieve linking of the process wells. Nine process wells have been successfully connected by RCL. The well spacing varied between 15 and $60 \mathrm{~m}$, and RCL at the Chinchilla UCG plant demonstrated linking speeds between $0.9 \mathrm{~m} /$ day and $11.3 \mathrm{~m} /$ day. The wide range of RCL speeds at Chinchilla, experienced in 
essentially the same geological conditions and similar coal quality, suggests that the speed is highly sensitive to the injection and production parameters. The maximal RCL speeds demonstrated at Chinchilla are the highest achieved in UCG operations on record.

We conducted systematic comparison of the calculated RCL speeds for various pressures and flow rates of the oxidant at the injection wells with the RCL speeds measured at Chinchilla. This work is still in progress and only preliminary results could be reported here. Figure 4 shows that the theory presented above gives a reasonable prediction of the RCL speeds measured in Chinchilla. The graph gives RCL speed in meters per day as a function of the oxidant mass flow. The parameters in calculations were selected according to: $A=6.2 \times 10^{11} s^{-1}, T_{E}=3 \times 10^{4} \mathrm{~K},\left(\phi_{g}\right)_{u}=0.15, T_{u}=25^{\circ} \mathrm{C}, T_{t} \rightarrow \infty$, polynomial approximations were used for evaluation of the enthalpies and $\lambda=\lambda(T)$ was taken from Ref.[4]. The crosses represent some of the empirical data obtained at Chinchilla. Further comparison with the results of field operation at Chinchilla and other sites will allow a broader validation of the presented theoretical model. One can see, however, that the experimental data from Chinchilla is reasonably represented by the calculations as per the described model.

\section{Conclusions}

This work introduces the theory of RCL that qualitatively explains and to a certain extent quantitatively models the salient features of the process observed in UCG operations. We explain the inevitability of a channel formation during RCL and demonstrate that consistent RCL is made possible by a combined contribution of several types of instabilities. We present an analysis of physical mechanisms that are responsible for stabilisation of the process. The results of this work explain why large scale UCG process based on reverse combustion would be highly impractical. The quantitative description of RCL is achieved by

- Analysis of the propagation speeds of oxygen-deficient, coal deficient and near-stoichiometric planar flames

- Analysis of the asymptotic structure and propagation speeds of flames that are curved and stretched (using the IDFE technique)

- Analysis of the hydrodynamic and pulsating stability of the different regimes of the flame

- Estimating the losses in narrow channels using the temperature loss equations obtained by the IDFE analysis

\section{Acknowledgments}

This work is supported by the Australian Research Council and by LINC Energy Ltd. 


\section{References}

[1] J.A. Britten and W.B. Krantz. Linear stability of planar reverse combustion in porous media. Comb. and Flame, 60:125-140, 1985.

[2] M.S. Blinderman and R. M. Jones. The Chinchilla IGCC project to date: Underground coal gasification and environment. In Proceedings of the 2002 Gasification Technologies Conference. San Francisco, October 27-30, 2002.

[3] T. J. Ohlemiller. Modeling of smoldering combustion propagation. Prog. Energy Combust. Sci., 11:277-310, 1985.

[4] J.A. Britten and W.B. Krantz. Asymptotic analysis of planar nonadiabatic reverse combustion fronts in porous media. Comb. and Flame, 101:151-161, 1986.

[5] D.A. Schult, B. J. Matkowsky, V. A. Volpret, and A.C. Fernandez-Pello. Propagation and extinction of forced opposed flow smolder waves. Comb. and Flame, 101:471-490, 1995.

[6] Y. Liu, M. Chen, J. Buckmaster, and T.L. Jackson. Smolder waves, smolder spots, and the genesis of tribrachial structures in smolder combustion. Proc. Comb. Inst., 30:323-329, 2005 .

[7] A. Y. Klimenko and A. G. Class. On premixed flames as gasdynamic discontinuities: A simple approach to derive their propagation speed. Combust. Sci. and Tech., 160:25-37, 2000 .

[8] A. Y. Klimenko and A. G. Class. Propagation of nonstationary curved flames with multistep reaction mechanisms. Combust. Sci. and Tech., 174:165-207, 2002.

[9] A. Y. Klimenko, A. G. Class, and S. M. O'Gorman. Near-equidiffusion disturbed premixed flames with wider reaction zones. Technical Report 2002/01, Dept. of Mech. Eng., The University of Queensland, 2002.

[10] A. G. Class, B. J. Matkowsky, and A. Y. Klimenko. A unified model of flames as gasdynamic discontinuities. J.Fluid Mech, 491:11-49, 2003.

[11] M.S. Blinderman and A. Y. Klimenko. Reverse combustion linking in underground coal gasification. In Proceedings of CHEMECA on CD ROM, paper (CC15) 160. Brisbane, 2005.

[12] A. G. Class, B. J. Matkowsky, and A. Y. Klimenko. Stability of planar flames as gasdynamic discontinuities. J.Fluid Mech, 491:51-63, 2003.

\section{A Stability analysis}

The variations of equations (3), (25), (26), (41) and (45) are given by

$$
\delta Q_{h}=\frac{Z_{Q}}{2} Q_{h}^{0} \delta T_{b}, \delta T_{b}=-T_{a}^{\prime} \frac{\delta \mu_{s}^{-}}{\left(\mu_{s}^{-}\right)^{0}}-\delta T_{l},
$$




$$
\begin{gathered}
\frac{\delta\left(\overline{m_{g}^{*}}\right)_{u}}{\left(\overline{m_{g}^{*}}\right)_{u}^{0}}+\frac{\delta H}{H^{0}}=\frac{\delta Q_{h}}{Q_{h}^{0}}, \delta H=\eta^{\prime} H^{0} \frac{\delta \mu_{s}^{-}}{\left(\mu_{s}^{-}\right)^{0}} \\
\frac{\delta\left(\overline{m_{s}^{*}}\right)_{u}}{\left(\overline{m_{s}^{*}}\right)_{u}^{0}}=\frac{\delta S}{S^{0}}, \frac{\delta\left(\overline{m_{g}^{*}}\right)_{u}}{\left(\overline{m_{g}^{*}}\right)_{u}^{0}}=\frac{\delta S+\delta U_{u}}{S^{0}+U_{u}^{0}}, \\
\frac{\delta \mu_{s}^{-}}{\left(\mu_{s}^{-}\right)^{0}}=\frac{\delta\left(\overline{m_{s}^{*}}\right)_{u}}{\left(\overline{m_{s}^{*}}\right)_{u}^{0}}-\frac{\delta\left(\overline{m_{g}^{*}}\right)_{u}}{\left(\overline{m_{g}^{*}}\right)_{u}^{0}}=\frac{1}{S^{0}+U_{u}^{0}}\left(\frac{U_{u}^{0}}{S^{0}} \delta S-\delta U_{u}\right)
\end{gathered}
$$

where

$$
T_{a}^{\prime} \equiv-\frac{\partial T_{a}}{\partial \ln \left(\mu_{s}^{-}\right)}, \quad Z_{Q} \equiv 2 \frac{\partial \ln \left(Q_{h}\right)}{\partial T_{b}}, \eta^{\prime} \equiv \frac{\partial \eta}{\partial \ln \left(\mu_{s}^{-}\right)}, \quad \eta \equiv \ln \left(H\left(\mu_{s}^{-}\right)\right)
$$

and $Z_{Q} \approx Z=T_{E} / T_{b}^{2} \gg 1$ (assuming $T_{b} \ll T_{t}$ ). Here, variations are considered small so that we use the leading order relationship $\overline{m_{i}^{*}}=\overline{m_{i}}=\phi_{i} \rho_{i} v_{i}$. The leading effect of the flame stretch, which is amplified by large $Z$, is in altering the burned temperature $T_{b}$. These equations result in

$$
B_{S} \delta S=B_{U} \delta U_{u}-B_{T} \delta T_{l}=B_{U} \delta U_{u}-B_{\gamma} \gamma_{0}-B_{\kappa} \frac{\kappa_{g}}{U_{u}^{0}}
$$

where

$$
\begin{gathered}
B_{S} \equiv \frac{Z_{Q}}{2} T_{a}^{\prime} \frac{U_{u}^{0}}{S^{0}}+\eta^{\prime} \frac{U_{u}^{0}}{S^{0}}+1, \quad B_{U} \equiv \frac{Z_{Q}}{2} T_{a}^{\prime}+\eta^{\prime}-1, \\
B_{T} \equiv \frac{Z_{Q}}{2}\left(S^{0}+U_{u}^{0}\right), \quad B_{\gamma}=B_{T} \theta_{s} T_{\Delta} l_{f}, \quad B_{\kappa}=B_{T} \theta_{g} T_{\Delta} l_{f}
\end{gathered}
$$

The value $\eta^{\prime}=0$ (and $T_{a}^{\prime}>0$ ) in oxygen-deficient flames, $\eta^{\prime}=1$ (and $T_{a}^{\prime}<0$ ) in carbon deficient flames and $0<\eta^{\prime}<1$ (and $T_{a}^{\prime} \sim 0$ ) in the near-stoichiometric flames; $\eta^{\prime}$ is linked to $T_{a}^{\prime}$ by the following equation

$$
T_{a}^{\prime}=\frac{\left(h_{s}^{(s)}\right)_{(\Delta)} \mu_{s}^{-}-\eta^{\prime} H}{\left(\overline{\mu C_{p}}\right)_{b}}
$$

\section{A.1 Unsteady effects in the preheat zone and pulsating stability}

Pulsating instability appears when a minor increase in the propagation velocity of a planar flame results in a change of the fuel/air ratio $\mu_{s}^{-}$that favours increase of the combustion temperature $\delta T_{b}$ and further speeds up the flame. We consider a planar flame and put $\gamma_{0}=0$ and $\kappa_{0}=0$. In the preheat zone, we obtain from (37)

$$
\frac{\partial \overline{\rho\left(h-h_{u}\right)}}{\partial t}+\frac{\partial \overline{m\left(h-h_{u}\right)}}{\partial x}-\frac{\partial}{\partial x}\left(\lambda \frac{\partial T}{\partial x}\right)=0
$$


Integration of this equation over $x$ from $-\infty$ to -0 (corresponding to the " $-r$ " location) results in

$$
\left(\overline{m\left(h-h_{u}\right)}\right)_{(-r)}=\left(\lambda \frac{\partial T}{\partial x}\right)_{(-r)}-\frac{d I_{h}}{d t}=Q_{h}-\frac{d I_{h}}{d t}
$$

where

$$
I_{h} \equiv \int_{-\infty}^{-0} \partial \overline{\rho\left(h-h_{u}\right)} d x
$$

Thus, unsteady effects result in the correcting term $d I_{h} / d t$ whose evaluation requires solving the system of equations

$$
\begin{gathered}
\frac{\partial \overline{\rho C_{p}}\left(T-T_{u}\right)}{\partial t}+\frac{\partial \overline{m C_{p}}\left(T-T_{u}\right)}{\partial x}-\frac{\partial}{\partial x}\left(\lambda \frac{\partial\left(T-T_{u}\right)}{\partial x}\right)=0 \\
\left(\overline{m_{g}^{*}}\right)_{u} H\left(\mu_{s}^{-}\right)=Q_{h}=\left(\lambda \frac{\partial T}{\partial x}\right)_{(x=-0)}
\end{gathered}
$$

Here we use the approximation of constant heat capacities. The boundary value of $T$ (that is $T_{b}^{0}$ in an undisturbed flame) tends to the quasi-steady value $T_{b}^{*}$ determined by the equation $T_{b}^{*}=T_{a}\left(\mu_{s}^{-}\right)$. The characteristic relaxation time is determined by reaction of equation (A.9) to a variation of $Q_{h}$ (that also causes variation of $\overline{m_{O 2}}$ and adjustment of the flame propagation speed) and can be assessed as

$$
\tau_{f} \approx \frac{\lambda_{r}\left(\overline{\rho C_{p}}\right)_{u}}{\left(\overline{m C_{p}}\right)_{u}^{2}}
$$

The simple relaxation equation

$$
\frac{d T_{b}}{d t}=\frac{T_{b}^{*}-T_{b}}{\tau_{f}} \Longrightarrow \frac{d\left(\delta T_{b}\right)}{d t}=\frac{\delta T_{b}^{*}-\delta T_{b}}{\tau_{f}}
$$

preserves the major properties of this behaviour. The variations

$$
\begin{gathered}
\delta Q_{h}=\frac{Z_{Q}}{2} Q_{h}^{0} \delta T_{b}, \delta T_{b}^{*}=-T_{a}^{\prime} \frac{\delta \mu_{s}^{-}}{\left(\mu_{s}^{-}\right)^{0}}, \frac{\delta\left(\overline{m_{g}}\right)_{u}}{\left(\overline{m_{g}}\right)_{u}^{0}}+\eta^{\prime} \frac{\delta \mu_{s}^{-}}{\left(\mu_{s}^{-}\right)^{0}}=\frac{\delta Q_{h}}{Q_{h}^{0}}, \\
\frac{\delta\left(\overline{m_{g}}\right)_{u}}{\left(\overline{m_{g}}\right)_{u}^{0}}=\frac{U_{u}^{0}}{S^{0}} \frac{\delta \mu_{s}^{-}}{\left(\mu_{s}^{-}\right)^{0}}, \quad \frac{\delta\left(\overline{m_{s}}\right)_{u}}{\left(\overline{m_{s}}\right)_{u}^{0}}=\frac{S^{0}+U_{u}^{0}}{S^{0}} \frac{\delta\left(\overline{m_{g}}\right)_{u}}{\left(\overline{m_{g}}\right)_{u}^{0}}
\end{gathered}
$$

are obtained from (A.1)-(A.4) for the case of a planar flame. Combining the equations results in

where

$$
\frac{d\left(\delta T_{b}\right)}{d t}=-\beta_{t} \frac{\delta T_{b}}{\tau_{f}}
$$

$$
\beta_{t}=\frac{Z_{Q}}{2} \frac{T_{a}^{\prime}}{S^{0} / U_{u}^{0}+\eta^{\prime}}+1
$$

The oxygen-deficient case is stable due to $T_{a}^{\prime}>0$ and $\eta^{\prime}=0$ while the carbon-deficient case is not since $T_{a}^{\prime}<0, \eta^{\prime}=1$ and $Z_{Q}$ is presumed large. The near-stoichiometric case $T_{a}^{\prime} \approx 0$ is stable up to the point where $\beta_{t}=0$ that is coincides with the point where $U_{u}$ reaches its maximum $\left(B_{S}=0\right.$ in (A.5)). 


\section{A.2 Harmonic disturbance and hydrodynamic stability}

The hydrodynamic instability appears when a planar flame changes its shape and forms a cavity on the coal surface. The cavity attracts an increased air flow that may further speed up the flame. In this section, we consider a planar flame with a small harmonic disturbance of the flame position. The undisturbed pressure and gas velocity are linked by Darcy's law $p^{0}=-U^{0} x^{*} / f$. We denote the streamwise and lateral coordinates by $x^{*}$ and $y^{*}$ where the asterisk is used to emphasise that the coordinates in use are Cartesian coordinates moving with the speed $S^{0}$ of the undisturbed planar flame. The position of the flame is given by $x_{f}^{*}=x_{f}^{*}\left(y^{*}, t\right)$ and we assume that the flame surface is harmonically disturbed

$$
\delta x_{f}^{*}=a \cos \left(k y^{*}\right)
$$

Upstream and downstream from the flame, the pressure

$$
p=p^{0}+\delta p=\left\{\begin{array}{cc}
-\frac{U_{u}^{0}}{f_{u}} x^{*}+a_{u} \frac{U_{u}^{0}}{f_{u}} \exp \left(k x^{*}\right) \cos \left(k y^{*}\right), & x^{*}<x_{f}^{*} \\
-\frac{U_{b}^{0}}{f_{b}} x^{*}+a_{b} \frac{U_{b}^{0}}{f_{b}} \exp \left(-k x^{*}\right) \cos \left(k y^{*}\right), & x^{*}>x_{f}^{*}
\end{array}\right\}
$$

satisfies $f \nabla^{2} p=\nabla \cdot(\mathbf{U})=0$ upstream and downstream from the flame (our consideration is applied to the regions just before and just after the flame but not within the flame or a long distance before the flame so that changes in density of the gas flow can be neglected). On the flame surface $x^{*}= \pm x_{f}^{*}$, we obtain to the leading order

$$
p=p^{0}+\delta p=\left\{\begin{array}{ll}
\left(\frac{U_{u}^{0}}{f_{u}}\left(a_{u}-a\right)\right) \cos \left(k y^{*}\right), & x^{*}=x_{f}^{*}-0 \\
\left(\frac{U_{b}^{0}}{f_{b}}\left(a_{b}-a\right)\right) \cos \left(k y^{*}\right), & x^{*}=x_{f}^{*}+0
\end{array}\right\}
$$

since in porous flows the pressure must be the same to the leading order on both sides of the flame we obtain

$$
\frac{U_{b}^{0}}{f_{b}}\left(a_{b}-a\right)=\frac{U_{u}^{0}}{f_{u}}\left(a_{u}-a\right)
$$

The gas velocity $U$ normal to the flame is given on the flame surface by

$$
U=U^{0}+\delta U=-f \frac{\partial p}{\partial x}=\left\{\begin{array}{ll}
U_{u}^{0}-a_{u} U_{u}^{0} k \cos \left(k y^{*}\right), & x^{*}=x_{f}^{*}-0 \\
U_{b}^{0}+a_{b} U_{b}^{0} k \cos \left(k y^{*}\right), & x^{*}=x_{f}^{*}+0
\end{array}\right\}
$$

while the stretch component $\kappa_{g}$ due to the gas flow divergence on the unburned side of the flame surface is evaluated by applying the operator $\nabla_{f}$ to the projection of $\mathbf{U}=-f \nabla p$ onto the flame surface

$$
\kappa_{g}=U_{u}^{0}\left(a_{u}-a\right) k^{2} \cos \left(k y^{*}\right)
$$

The amplitudes $a_{u}$ and $a_{b}$ are linked by the equation

$$
\frac{-a_{b} U_{b}^{0}}{a_{u} U_{u}^{0}}=\frac{\delta U_{b}^{0}}{\delta U_{u}^{0}}=B_{b} \frac{U_{b}^{0}}{U_{u}^{0}} \Longrightarrow a_{b}=-B_{b} a_{u}
$$


where the coefficient $B_{b}$ is determined by the kinetics of the flame. Equations (A.14) and (A.16) result in

$$
a_{u}=B_{a} a, B_{a} \equiv \frac{1-B_{f}}{1+B_{f} B_{b}}, B_{f} \equiv \frac{U_{b}^{0}}{f_{b}} \frac{f_{u}}{U_{u}^{0}}
$$

With exception of the vicinity of the stoichiometric point, the coefficient $B_{b}$ is very close to unity (although not exactly 1 since temperature and composition of the products experience some changes when $U_{u}$ varies). In the vicinity of the stoichiometric point the exact value of $B_{b}$ is not important since $f_{b} \gg f_{u}$ and $B_{f} \approx 0$ due to full (or nearly full) consumption of the coal under this regime. Hence, we can put $B_{b}=1$. The curvature, the gas-phase stretch and the upstream gas velocity close to the flame are now given by

$$
\begin{gathered}
\gamma_{0}=\frac{\partial^{2} \delta x_{f}}{\partial y^{2}}=-a k^{2} \cos (k y), \kappa_{g}=-B_{2} U_{u}^{0} a k^{2} \cos \left(k y^{*}\right) \\
\delta U_{u}=-a B_{a} U_{u}^{0} k \cos (k y), B_{2} \equiv \frac{2 B_{f}}{1+B_{f}}
\end{gathered}
$$

Substitution of these equations into (A.5) results in

$$
B_{S} \delta S=\left(-B_{U} B_{a} U_{u}^{0} k+\left(B_{\gamma}+B_{\kappa} B_{2}\right) k^{2}\right) a \cos (k y)
$$

Since the fame moves in the negative direction $d\left(\delta x_{f}\right) / d t=-\delta S$ and

$$
\frac{d a}{d t}=a \beta_{a}, \beta_{a}=\frac{B_{U} B_{a} U_{u}^{0}}{B_{S}} k-\left(B_{\gamma}+B_{\kappa} B_{2}\right) \frac{k^{2}}{B_{S}}
$$

The flame becomes hydrodynamically stable in the vicinity of maximum of $S$ where $B_{U}=$ 0 , and remains stable in the region of increasing $U_{u}$ and decreasing $S$ where $B_{S}>0$ and $B_{U}<0$. The hydrodynamic and pulsating stability is lost at the point of maximum $U_{u}$ where $B_{S}=0$. The oxygen-deficient and carbon-deficient regions are hydrodynamically unstable; for these regimes, assuming the $Z_{Q} T_{a}^{\prime}$ is large, we can write

$$
\frac{\delta S}{S^{0}}=\frac{\delta U_{u}}{U_{u}^{0}}-\frac{\delta T_{l}}{T_{a}^{\prime}}, \quad \beta_{a}=S^{0} k\left(B_{a}-\frac{\left(\theta_{s}+\theta_{g} B_{2}\right) T_{\Delta}}{T_{a}^{\prime}} l_{f} k\right)
$$

Hence the scale $l_{\beta}=1 / k_{\beta}$ of the most unstable mode is given by

$$
l_{\beta}=2 \frac{\left(\theta_{s}+\theta_{g} B_{2}\right) T_{\Delta}}{B_{a} T_{a}^{\prime}} l_{f}
$$




\section{FIGURE CAPTIONS}

Figure 1. Schematic of Reverse Combustion Linking (RCL)

Figure 2. Dependence of the temperature of the products on the coal/air mass ratio. Dashed line - irreversible reactions; solid line - more realistic reactions.

Figure 3. Dependence of the flame propagation speed on the speed of supplied air; a) qualitative dependence, b) dependence calculated for the conditions used in Section 6 . The lines as in Figure 2.

Figure 4. The speed of RCL as function of supplied air rate. Solid line - theory; symbols - data from RCL in Chinchilla [11]. 


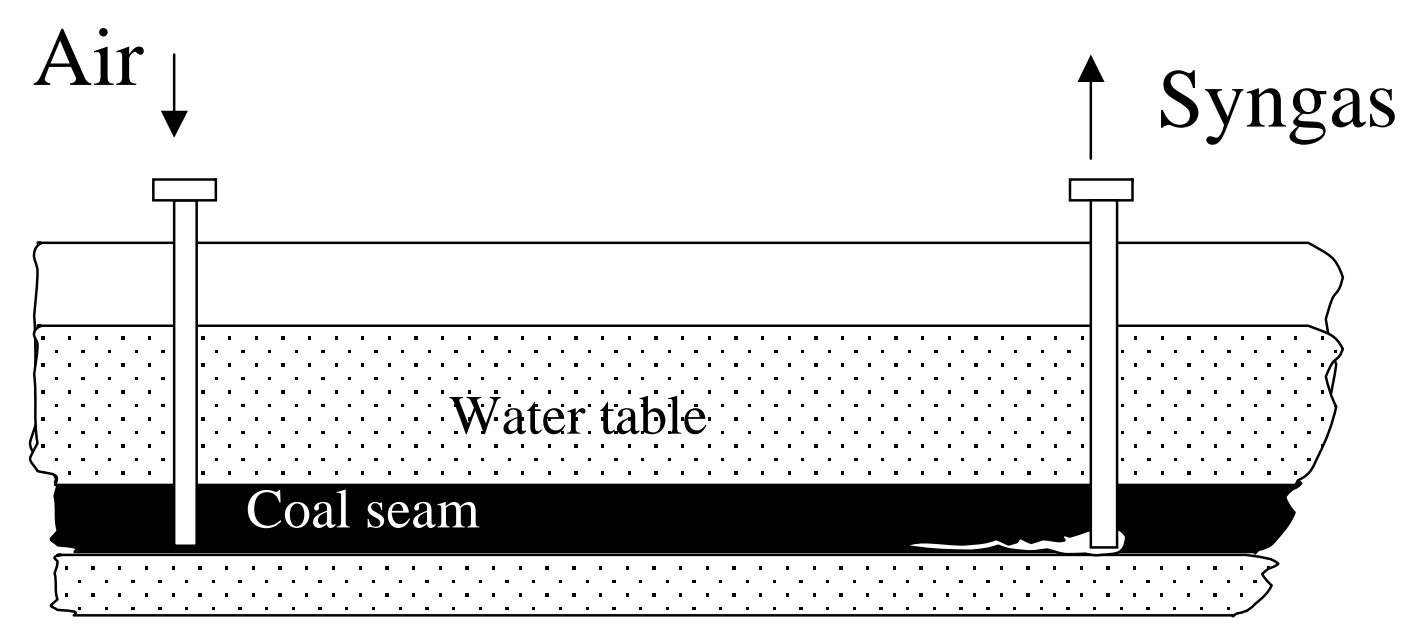




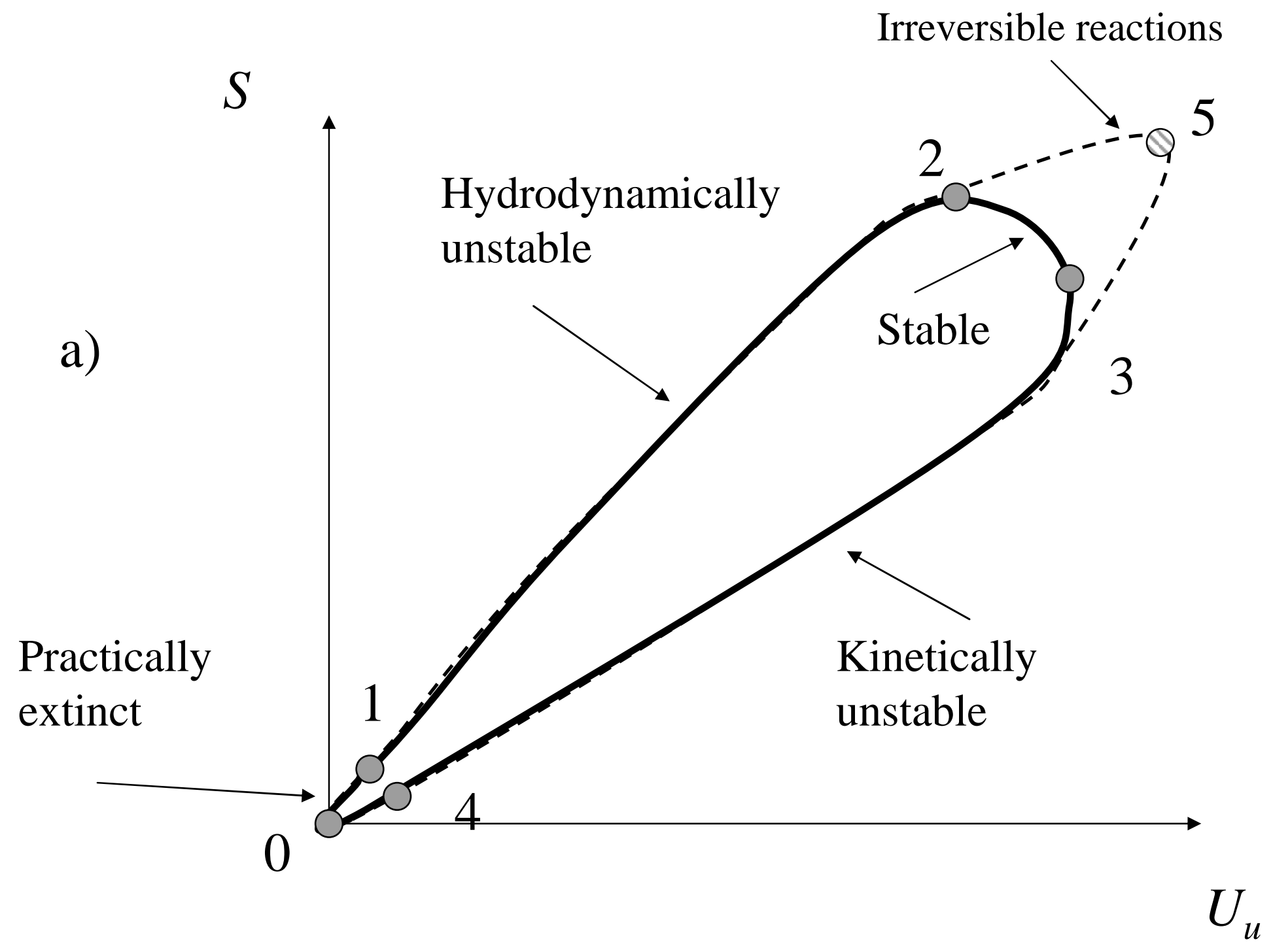




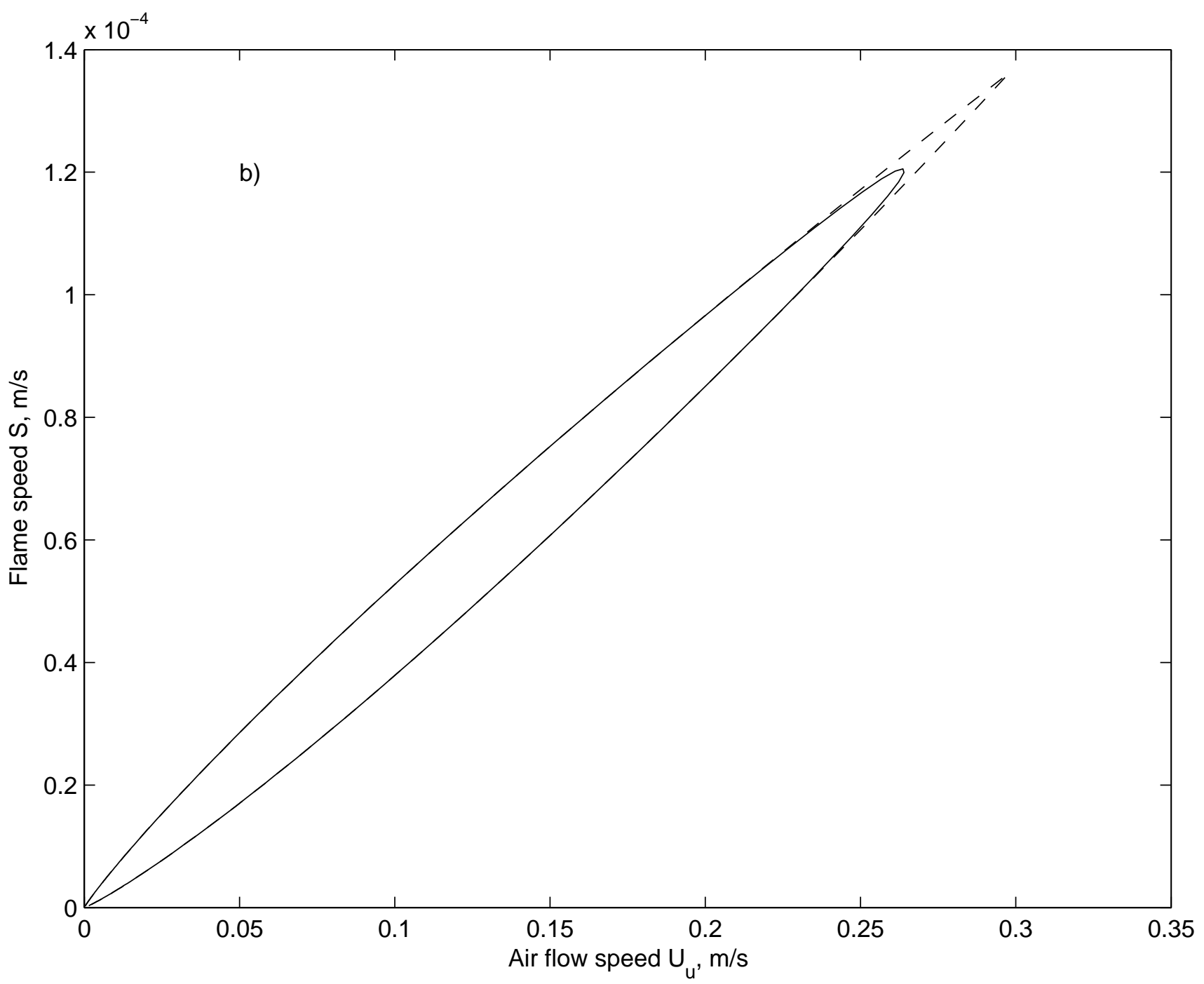




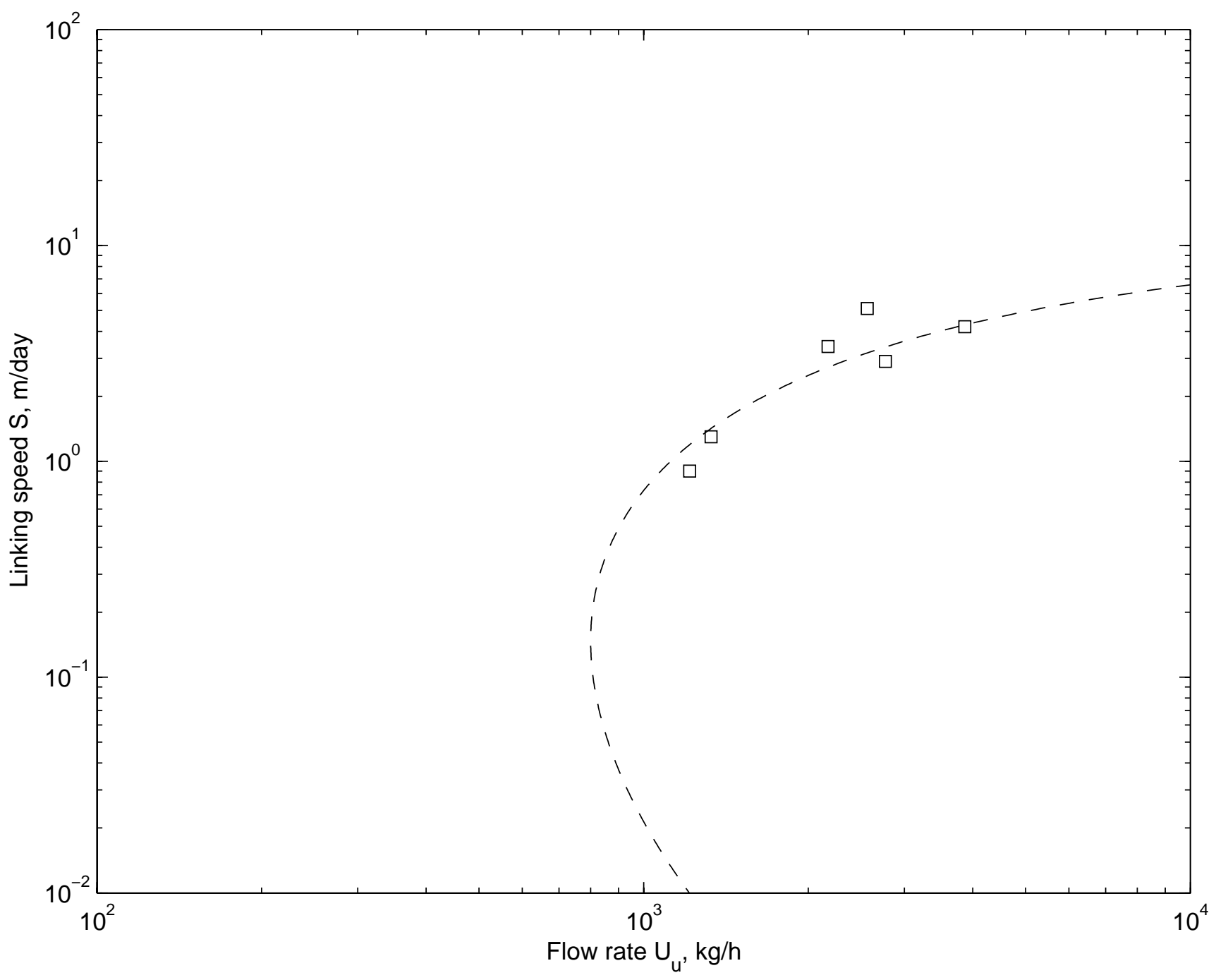

